\title{
FMRI and EEG Reactions to Hand Motor Tasks in Patients with Mild Traumatic Brain Injury: Left-Hemispheric Sensitivity to Trauma
}

\author{
Ludmila Zhavoronkova1*, Sofia Moraresku1,2, Galina Boldyreva1, Elena Sharova1, \\ Svetlana Kuptsova ${ }^{1,3}$, Alexander Smirnov ${ }^{4}$, Eugen Masherov ${ }^{4}$, Olga Maksakova ${ }^{4}$, Igor Pronin ${ }^{4}$ \\ ${ }^{1}$ Institute of Higher Nervous Activity and Neurophysiology, Russian Academy of Sciences, Moscow, Russia \\ ${ }^{2}$ Lomonosov Moscow State University, Moscow, Russia \\ ${ }^{3}$ Center for Speech Pathology and Neurorehabilitation, Moscow, Russia \\ ${ }^{4}$ N.N. Burdenko National Medical Research Center of Neurosurgery, Moscow, Russia \\ Email: ^1zhavoronkova@hotmail.com
}

How to cite this paper: Zhavoronkova, L., Moraresku, S., Boldyreva, G., Sharova, E., Kuptsova, S., Smirnov, A., Masherov, E., Maksakova, O. and Pronin, I. (2019) FMRI and EEG Reactions to Hand Motor Tasks in Patients with Mild Traumatic Brain Injury: Left-Hemispheric Sensitivity to Trauma. Journal of Behavioral and Brain Science, 9, 273-287.

https://doi.org/10.4236/jbbs.2019.96020

Received: May 14, 2019

Accepted: June 27, 2019

Published: June 30, 2019

Copyright ( 2019 by author(s) and Scientific Research Publishing Inc. This work is licensed under the Creative Commons Attribution International License (CC BY 4.0).

http://creativecommons.org/licenses/by/4.0/ (c) (i) Open Access

\begin{abstract}
Background: Mild traumatic brain injury (mTBI) is one of the most common forms of cerebral pathology in young people and disorders involve dysfunctions in cognitive and motor spheres. We would like to examine the structural and functional alterations of the brain in patients with mTBI while performing hand movements. Methods: Twenty healthy right-handed subjects (age $25.1 \pm 3.9$ ) and 10 patients (age $27.9 \pm 7.3$ ) with $\mathrm{mTBI}$ without hemiparesis participated in the study using functional magnetic resonance imaging (fMRI) and electroencephalography (EEG). FMRI and EEG reactions were analysed during right- and left-hand movements. Results: It was shown that fMRI reactive changes have a larger inter-individual variability of activation during left-hand movements in comparison with right-hand ones in healthy subjects. The TBI patients demonstrated an increase of a diffuse component of fMRI reactive changes compared to healthy people. A greater number of the brain structures was involved, mainly at the subcortical level, mostly in the left hemisphere during right-hand movement. EEG study demonstrated coherence changes for the slow (delta) frequency bands in the left hemisphere, while performing both hand movements. In healthy persons, EEG coherence changes were observed in the fast (alhpa2) frequency band predominantly in contralateral hemispheres, while performing hand movements. Conclusion: So, fMRI and EEG studies revealed the most expressed pathological reactive changes in the left hemisphere and the brain cortical structures during right-hand movements in patients after mTBI. These data allowed us to propose that the younger brain structures were the most sensitive to mTBI.
\end{abstract}




\section{Keywords}

fMRI, EEG, Traumatic Brain Injury, Right- and Left-Hand Movements

\section{Introduction}

Mild traumatic brain injury (mTBI) is one of the most common forms of cerebral pathology occurring in adolescence and young people [1] [2] [3]. Posttraumatic disorders are typically multi-component and involve the damage of pathways, cortical and subcortical structures [2] [4] [5]. MTBI causes structural and functional alterations to the brain. Diffuse axonal injury is frequent in TBI, and disintegration is a significant pathogenetic factor of TBI and mTBI [1] [2] [4] [5] [6] [7]. Disintegration processes in the genu and body of the corpus callosum were described mostly in patients with mTBI. Some of these changes are thought to be progressive in nature, and potentially increase the risk for early cognitive decline and dementia observed in mTBI patients [8].

Over the past decades, many publications have appeared on the links between observable neuropsychological and behavioral symptoms of mTBI using clinically available brain imaging techniques [9] [10] [11]. Increased diffusivity as evidenced with an apparent diffusion coefficient in both left and right dorsolateral prefrontal cortex and subcortical structures was observed very often in mTBI patients [11] [12]. Results of these investigations allowed the authors to propose accelerated brain aging and demonstrate physical decline, cognitive impairments, and brain volume loss after trauma [10] [11] [12] [13] [14]. The authors hypothesized that the discrepancy between chronological and predicted brain age would be reflected in cognitive changes and consistent with age-related cognitive impairment in older individuals [15].

Advances in brain imaging methodology have revealed important information regarding both structural [1] [2] [16] [17] and functional [18] [19] [20] [21] alteration after mTBI. Patients with mTBI had a more disperse brain activation pattern with additional increases in activity outside of the regions of interest (ROIs) as revealed by fMRI blood oxygen level dependent signals. fMRI demonstrates blood oxygenation changes in activated brain regions during task execution, thereby depicting the exact level of engagement of different cerebral structures in function implementation [14] [20] [21] [22]. Even in the absence of apparent neurological signs, patients may experience various long-term post-injury problems, e.g., executing professional activities [17] [18] [23] [24]. However, neurophysiological mechanisms of these disorders have not been studied sufficiently and require special research.

FMRI studies that have included healthy individuals, thereby expanding our knowledge of structures involved in reactive brain rearrangements during execution of various tests, may be helpful in preventing impaired brain function after injury [25] [26] [27] [28]. Our previous studies analysing hemodynamic 
brain reactions to motor tasks in healthy people showed the greatest locality and reproducibility of fMRI responses during fist clenching in comparison with other motor tasks, such as fingerpicking and others [26] [27]. That is why this paradigm of motor task was used as the most appropriate in mTBI patients.

EEG study using coherence measures is a highly informative approach to estimate the reactive changes in the human brain. Analysis of EEG coherence allows us to evaluate the degree of functional connectivity between different regions of the brain [29] [30] [31]. This method of EEG analysis is informative and successfully used in studying higher mental, motor, and other functions in healthy individuals and patients with different forms of cerebral pathology including TBI [18] [19] [24] [25] [26] [29] [30] [31].

Combination of fMRI and EEG results provides the most complete analysis of structural-functional cerebral damage [32]-[38]. FMRI reflects changes in the blood oxygenation level in activated brain areas, supplying data for a complete analysis of structural rearrangements. EEG contains a temporal component of the functional brain activation. It is the best approach for neurophysiological estimation of structures involved in brain-reactive processes. Using fMRI and EEG together offers new opportunities to study impaired functions in patients with mTBI [32]-[38].

Many studies have been devoted to the specificity of fMRI and EEG reactions to hand motor tasks in patients with hemiparesis [33] [36] [37] [38]. An analysis of PMRI and EEG activation in mTBI patients without hemiparesis presents a particular interest and represents an adequate model for studying the dynamics of function restoration after brain trauma. Furthermore, such analysis provides understanding of basic mechanisms of human brain functioning. The aim of our study was to examine the specificity of reactive patterns provided by fMRI and EEG data in patients with $\mathrm{mTBI}$, while performing movements with the right and left hand, compared to healthy subjects with special analysis of hemispheric sensitivity to trauma.

\section{Materials and Methods}

\section{Participants and Procedure}

The study included 20 healthy subjects ( 11 men and 9 women; aged $25.1 \pm 3.9$ years). The group of patients included 10 subjects with mTBI (6 men and 4 women, aged of $27.9 \pm 7.3$ years). The inclusion criteria for the group of patients were the absence of hemiparesis and local lesions in the sensorimotor cortex or other areas of the brain. Three patients of the group had more MRI signs of traumatic changes in the left hemisphere; three patients, in the right hemisphere; four other patients, bilaterally. The functional test was separate right and left-hand clenching-unclenching with the eyes closed. fMRI data were recorded according to the block paradigm consisting of alternating rest and motor testing periods of $30 \mathrm{~s}$ each. The results of five tests were averaged. EEG recording included a similar procedure and was performed after fMRI study. According to the Annett 
Hand Preference Questionnaire, all healthy subjects and patients were right-handed. Each subject signed informed consent for the study approved by Ethics Committee at Institute of Higher Nervous Activity and Neurophysiology of Russian Academy of Sxciences.

\section{1) FMRI recording and analysis}

The fMRI study was performed using a General Electric Signa HDxt MRI scanner (United States) with a magnetic field of $3 \mathrm{~T}$. fMRI data were processed using the SPM8 software based on Matlab R2014a both individually and in a group. Movement artifacts were corrected based on the generalized linear model (GLM). Statistical thresholds at the voxel level $\mathrm{p}<0.001$ (unc.) were used to construct individual activation maps with a corrected cluster significance level $\mathrm{p}$ (FWE-corr) $<0.01$. The data were averaged for a group using one-sided one-sample t-test. A two-sample t-test was used to compare independent samples with each other. We used statistical thresholds at the voxel level $\mathrm{p}<0.001$ (unc.) with a corrected cluster significance level $\mathrm{p}$ (FWE-corr) $<0.05$ to analyse activation maps separately for each group. When comparing groups of TBI patients and healthy subjects, voxel threshold corresponded to $\mathrm{p}<0.001$ (unc.) with a corrected cluster significance level $\mathrm{p}$ (FWEcorr) $<0.01$. The activated areas were verified and located (MNI-coordinates) identifying their activation volume in voxels (Vox) with the Automated Anatomical Labeling (AAL) application based on Matlab R2014a [16]. The structures in resulting tables were combined into larger structural and functional units. For example, the motor area included the gyrus precentralis and paracentral lobule; the frontal area included frontal sup., supramarginal, frontal mid., etc. Brain activation volumes were compared between healthy subjects and TBI patients by Statistica 6.0 software and Student's t-test. In case of significant changes, the normalised increase in volumes (\%) was estimated in patients compared to healthy subjects.

\section{2) EEG recording and analysis}

EEG was recorded at rest state and executing hand movement tasks using $\mathrm{Ni}$ hon Cohden equipment (Japan). The electrical activity from the scalp was recorded at 19-cites: Fp1, Fz, Fp2, F3, F4, F7, F8, C3, Cz, C4, P3, Pz, P4, O1, O2, T3, T4, T5, T6 according to the international $10-20$ system. The ground electrode was located at Fz with electrode impedance lower than 5. Each EEG trace lasted at least $60-70 \mathrm{~s}$ and was recorded using a sampling frequency of $100 \mathrm{~Hz}$. The filters were set to $35 \mathrm{~Hz}$ with bandwidth of $0.5-30 \mathrm{~Hz}$. For further analysis we use artifact-free segments of monopolar EEG recordings with indifferent ear electrodes, while rejecting segments with eye blinks and artifacts. The last $50-60 \mathrm{~s}$ of EEG segmented in epochs of $5 \mathrm{~s}$ (10 epochs) were subjected for Fast Fourier. The last 45 - $50 \mathrm{~s}$ of EEG were segmented in epochs of $5 \mathrm{~s}$ (a total no less than 10 epochs were taken). EEG power and coherence were calculated for the following frequency bands: delta-2 - $3.9 \mathrm{~Hz}$; theta-4.39 - 7.8 Hz; alpha1-8.2 - $10.2 \mathrm{~Hz}$; alpha-10.5 $12.9 \mathrm{~Hz}$; beta-13.3 - 30.1 Hz. Coherent indicators were calculated as averaged for each frequency range, squared absolute values of the complex spectral coherence 
using the program MatLab 6.0 of Math Works Inc. We use the Mann-Whitney test to compare independent groups, i.e. healthy subjects and patients, and the Wilcoxon test for linked sets.

\section{Results}

\subsection{Results of fMRI Study}

Group analysis of fMRI data in healthy persons is presented in Figure 1(A) and Figure 1(B). FMRI response changes during right-hand movement (Figure 1(A)) demonstrated the activation of the contralateral, left sensorimotor cortex in the precentral and postcentral gyri, a supplementary motor area in the medial parts of the superior frontal gyrus, and ipsilateral hemisphere of cerebellum.

An analysis of group mean fMRI responses to left-hand movement (Figure (B)) revealed only one main activation area in the sensorimotor area of the contralateral right hemisphere. This phenomenon can be explained by the fact, that individual fMRI responses while moving non-dominant left hand are more variable than those with right-hand movement. Some subjects have no activation area in the cerebellum ipsilateral hemisphere, while the sensorimotor cortex of the ipsilateral hemisphere and supplementary motor area are activated in others.

Noteworthy, activation areas in most individual fMRI responses were in the ipsilateral hemisphere of the cerebellum but their exact location and size differed (Figure 2).

The inter-group comparison of fMRI reactions (patients and healthy subjects) revealed the greatest differences in moving the dominant right hand (Figure 3(A)). A diffuse component of fMRI responses was present in patients, while
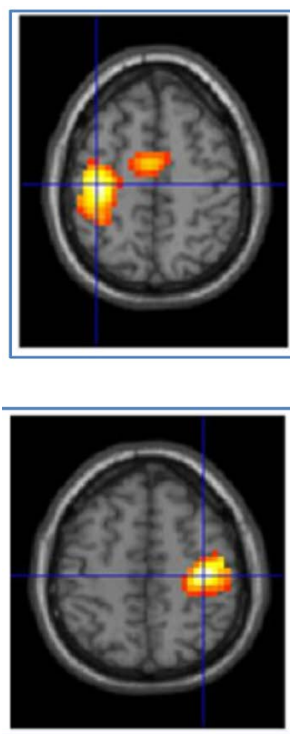

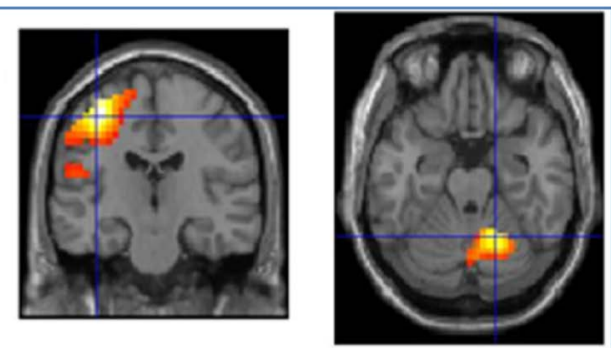

(A)

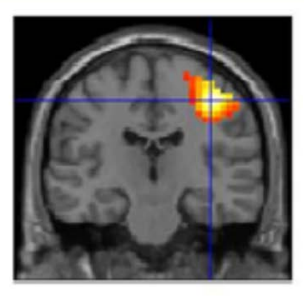

(B)

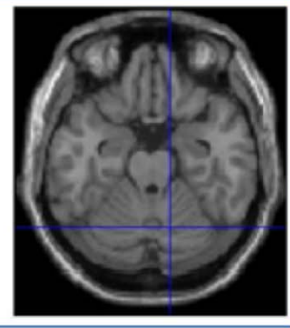

Figure 1. Group-average fMRI responses for healthy subjects during (A) right-hand movement and (B) left-hand movement $(\mathrm{n}=20, \mathrm{t}=3.53, \mathrm{p}(\mathrm{unc})<0.001$, with cluster correction for multiple comparison $\mathrm{p}(\mathrm{FWE}-\mathrm{corr})<0.05)$. 
Healthy subject Sch.

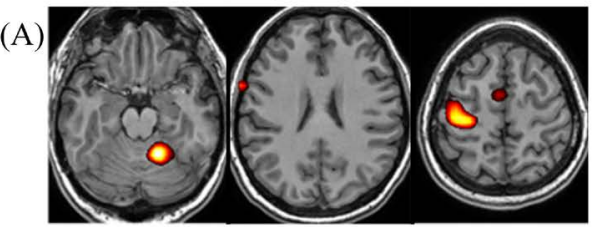

(B)

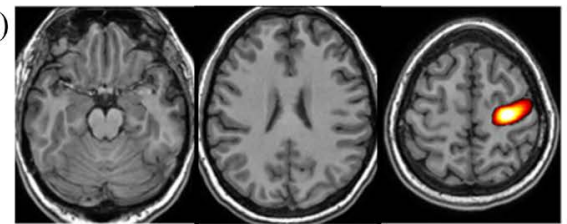

Healthy subject G.
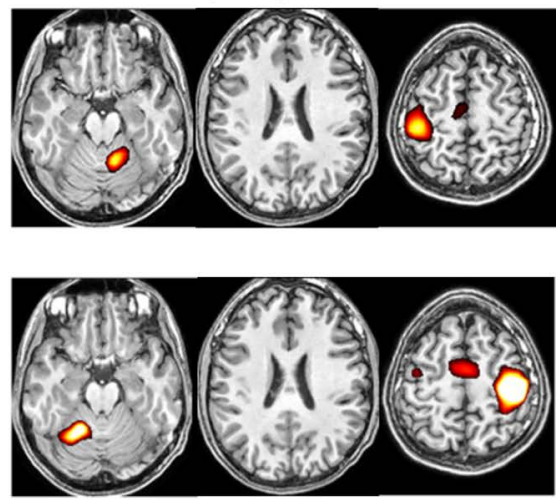

Figure 2. Examples of individual fMRI responses during right-hand (A) and left-hand movement (B) in healthy subjects Sch. and G.

(A)

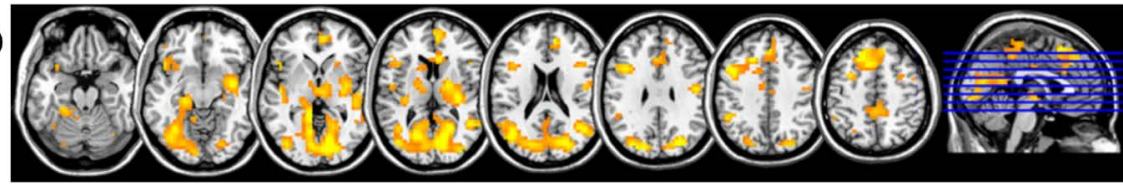

(B)

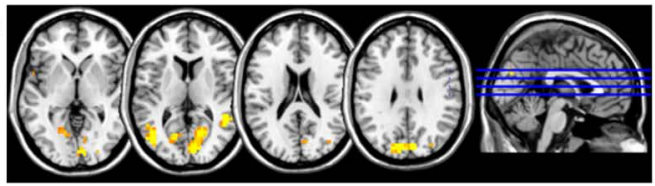

Figure 3. Comparison of fMRI responses during (A) right-hand movement and (B) left-hand movement between a group of TBI patients and healthy subjects ( $\mathrm{p}$ (unc) $<0.001$, with cluster correction for multiple comparisons $\mathrm{p}$ (FWE-corr) $<0.01$, patient group $>$ group of healthy subjects).

responses were more local in healthy subjects. Moreover, the subcortical structures and various nonspecific cortical areas of the contralateral hemisphere were significantly more involved in responses in TBI patients, which is not common for the motor response of healthy subjects. In general, the left-hand movement caused no significant differences in fMRI responses between the groups (Figure $3(B)$ ). However, there were slight differences in the occipital cortex and subcortical structures. Furthermore, the fMRI activation volume was slightly higher in the patient group.

An analysis of group mean fMRI responses to left-hand movement (Figure 1(B)) revealed only one main activation area in the sensorimotor area of the contralateral right hemisphere. This phenomenon can be explained by the fact, that individual fMRI responses while moving non-dominant left hand are more variable than those with right-hand movement. Some subjects have no activation area in the cerebellum ipsilateral hemisphere, while the sensorimotor cortex of the ipsilateral hemisphere and supplementary motor area are activated in others.

Individual fMRI responses in the TBI group presented high inter-individual variation, but all the patients had the diffuse form of brain response, in contrast 
to the healthy group with a more expressive response to right-hand movement $s$ (Figure 4(A)). The execution of this task in patients involved a larger number of cerebral structures compared to the norm; this effect manifested itself in both hemispheres (Figure 4(B)). Less distinctive features were observed in fMRI responses to left-hand movement in patients with TBI compared to healthy subjects (Figure 4(A), Figure 4(B)).

The statistical analysis of fMRI in different brain structures also showed the greatest response to right-hand movement in the left hemisphere. Activation volume in the motor cortex, supplementary motor area, and vermis was much larger in patients than in healthy subjects. Moreover, the subcortical nuclei, thalamus, limbic system, and some areas of cerebral cortex, nonspecific for the motor analyzer in healthy subjects (temporal and occipital) were more involved in patients (Figures $5(\mathrm{~A})(\mathrm{I})$ ). In contrast, fMRI response slightly increased in many structures of the ipsilateral hemisphere but these differences were insignificant in patients (Figures $5(B)(I)$ ). Left-hand movement led to insignificant changes covering more brain areas in patients compared to the norm; fMRI activation volume significantly increased only in the occipital regions of both hemispheres (Figure 5(II)).

We estimated the degree of changes in patients with TBI compared to healthy subjects and the normalized increase of fMRI activation volume in different areas of the cortex and subcortical structures. It was shown that patients have had a more expressive fMRI change in the subcortical structures and nonspecific cortex areas than in the cortical projection areas. During right-hand movement, fMRI activation volume in the motor and supplementary motor areas of the contralateral hemisphere increased by $20 \%$ and $33 \%$, respectively, in the TBI group compared to the control group, and fMRI activation volume in the nonspecific cortical areas (temporal and occipital) and subcortical structures increased. In left-hand movement, the activation volume of occipital fMRI responses in the contralateral and ipsilateral hemisphere increased by $80 \%$ and $70 \%$, respectively, in patients with TBI (Table 1 ).
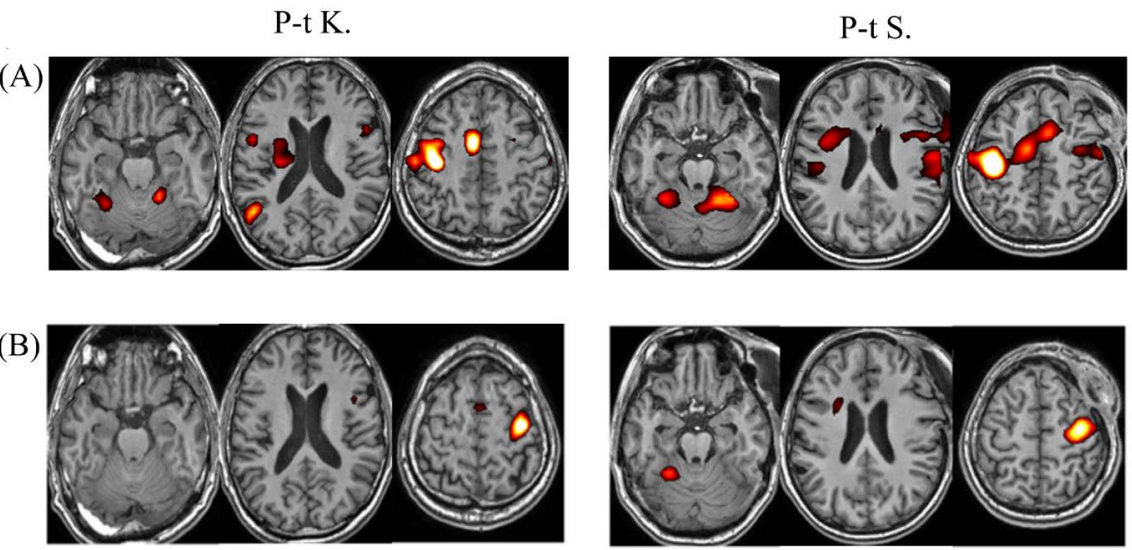

Figure 4. Examples of individual fMRI responses during right-hand movement (A) and left-hand movement (B) in P-t K. and P-t S.

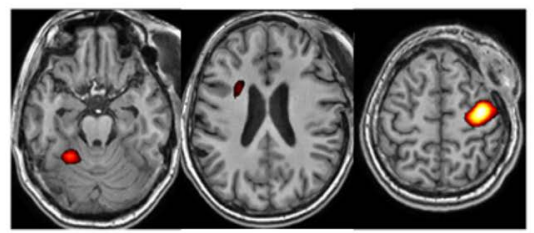


Table 1. Comparison of fMRI response volumes in different brain structures (in voxels) according to Student's test between healthy subjects and patients with TBI (differences at the 5\% level are shown).

\begin{tabular}{|c|c|c|c|c|c|}
\hline Brain region & Mean for healthy subjects & Mean for patients & $\mathrm{t}$ & $\mathrm{p}$ & $\begin{array}{l}\text { Normalized volume } \\
\text { increase in } \%\end{array}$ \\
\hline \multicolumn{6}{|c|}{ In the right-hand movement } \\
\hline Motor cortex (contralateral) & 118.6375 & 166.5468 & -2.07764 & 0.04670 & 20 \\
\hline Supplementary motor area (contralateral) & 23.0411 & 46.8741 & -2.11152 & 0.043464 & 33 \\
\hline Occipital cortex (contralateral) & 7.2726 & 55.8213 & -2.39719 & 0.023187 & 70 \\
\hline Temporal cortex (contralateral) & 8.0861 & 57.6505 & -2.68583 & 0.011844 & 70 \\
\hline Cerebellar vermis & 15.9089 & 52.0101 & -2.28924 & 0.029534 & 50 \\
\hline Thalamus (contralateral) & 3.8184 & 28.7676 & -2.31113 & 0.028132 & 75 \\
\hline Subcortical nuclei (contralateral) & 1.5001 & 45.4359 & -2.34546 & 0.026054 & 90 \\
\hline Limbic system (contralateral) & 8.1834 & 46.7075 & -2.38106 & 0.024048 & 70 \\
\hline \multicolumn{6}{|c|}{ In the left-hand movement } \\
\hline Occipital cortex (contralateral) & 1.4539 & 9.3351 & -2.52321 & 0.017367 & 80 \\
\hline Occipital cortex (ipsilateral) & 2.6352 & 14.6665 & -2.39777 & 0.023156 & 70 \\
\hline
\end{tabular}

Note: bold type is demonstrated the highest percent of fMRI volume's changes.

I
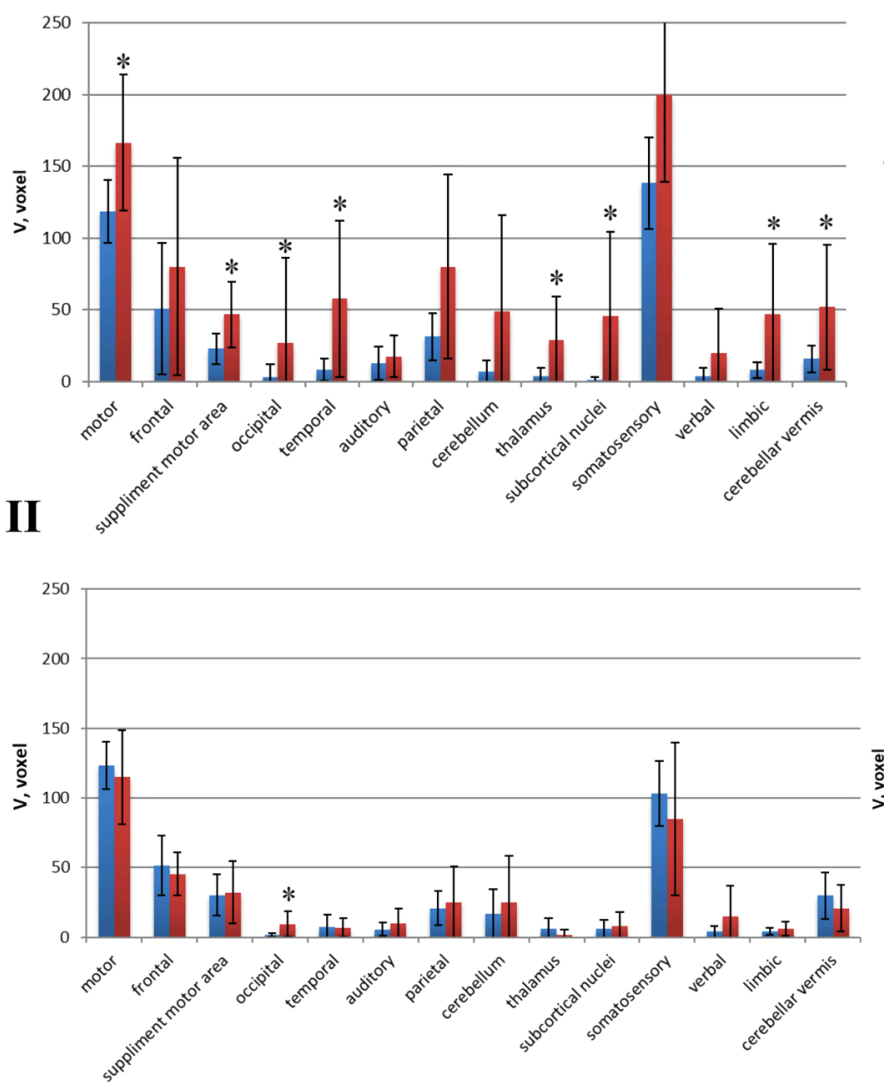

(B)
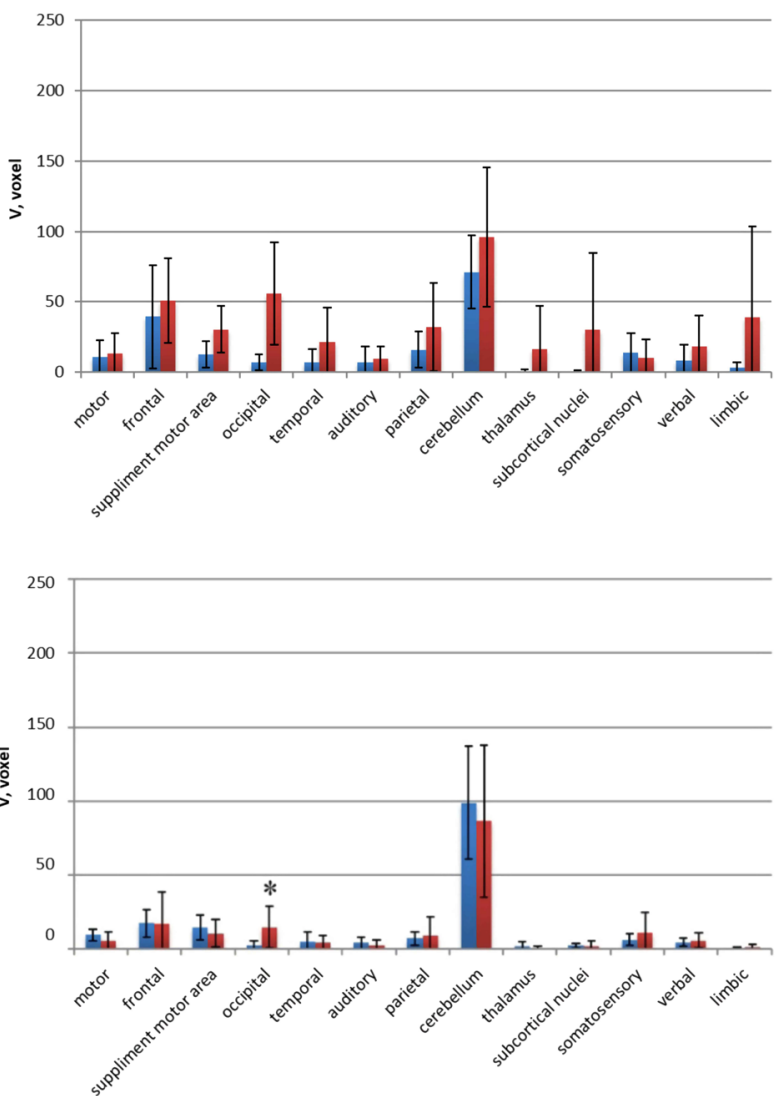

Figure 5. Statistical analysis of fMRI activation volume of different brain structures in healthy subjects and TBI patients. I-mean volumes of fMRI responses in the right-hand movement; II-mean volumes of fMRI responses in the left-hand movement; A-contralateral hemisphere; B-ipsilateral hemisphere; ${ }^{*} \mathrm{p}<0.05$. Blue bars-healthy subjects; red bars-patients with mTBI. 


\subsection{Results of EEG Study}

In healthy persons, EEG reactive changes associated with hand movement compared to baseline activity were more variable than fMRI responses. Figure 6 shows a group mean of reactive changes during right (A) and left-hand movements (B). EEG analysis demonstrates an increased coherence of oscillations (alpha2 frequencyband) and a greater one in the left hemisphere (on the side of the main fMRI response), while performing right-hand movements. In most cases, these changes were limited to the sensorimotor zone, i.e. central-frontal-temporal areas. It is important to note that, in motor tests, increased EEG coherence in the fast frequency alpha-band was accompanied by a decrease of coherence in the slow frequency bands (delta-, theta- and alpha1). Reactive changes had less local hemispheric specificity for left hand performance than coherence changes due to operations with the right hand.

The averaged changes of EEG coherence in baseline state for TBI patients compared to the healthy group, demonstrating a global decrease of EEG coherence for different frequency bands in patients, are presented in Figure 7. The averaged reactive changes of EEG coherence in right-hand and left-hand movement are shown in Figure 7.

EEG analysis demonstrates an increased coherence of slow frequency bands in the left hemisphere for both right and left-hand movements. There were reactive changes in the delta-band as right-hand movements were performed; an increased EEG coherence in the delta and theta band were observed, with left hand movement.

\section{Discussion}

The results of our study demonstrate fMRI reactive changes at specific ROI induced by hand motor tasks in healthy persons and agree with others' research

(A)

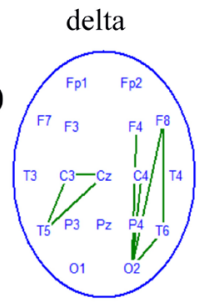

(B)

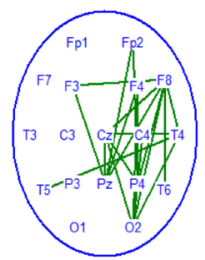

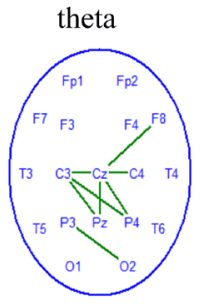
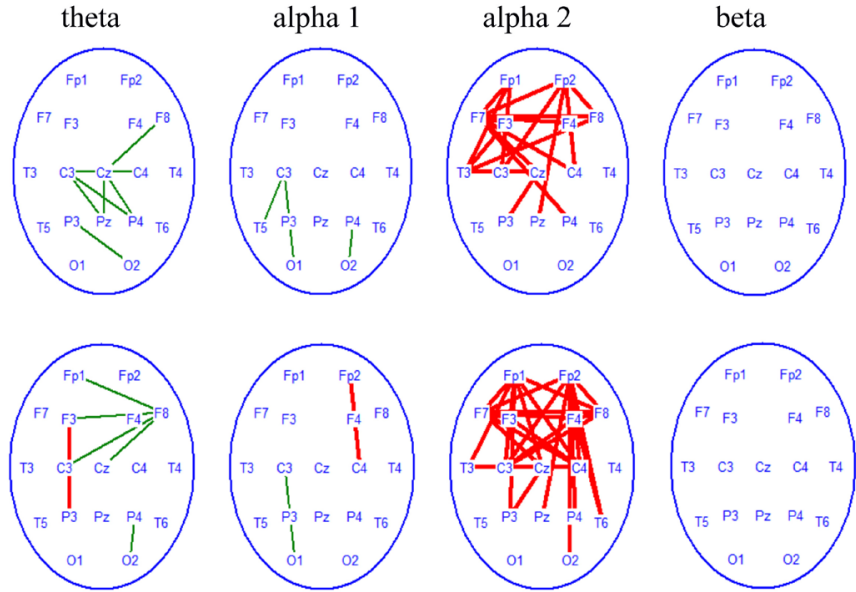

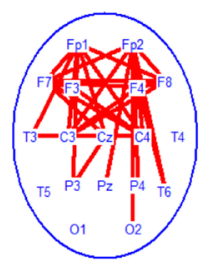

Figure 6. Averaged changes of EEG coherence while performing right-hand movement (A) and left-hand $(B)$ movement in healthy persons $(n=20)$ compared to baseline state $(p<0.01)$. Red lines demonstrate an increase of EEG coherence during hand movements, while green lines demonstrate a decrease of EEG coherence. 
(A)
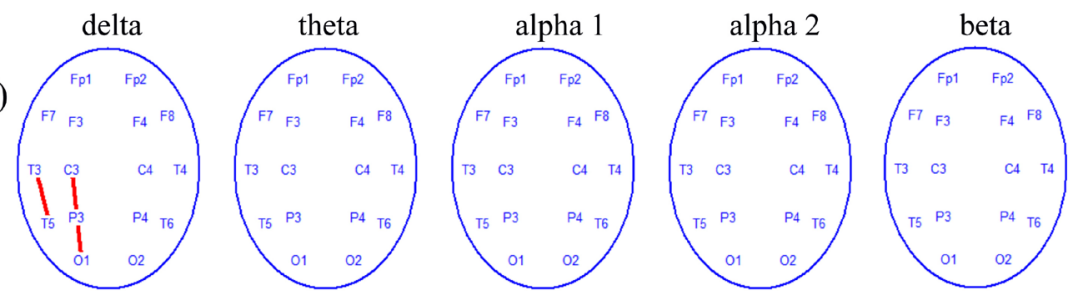

(B)
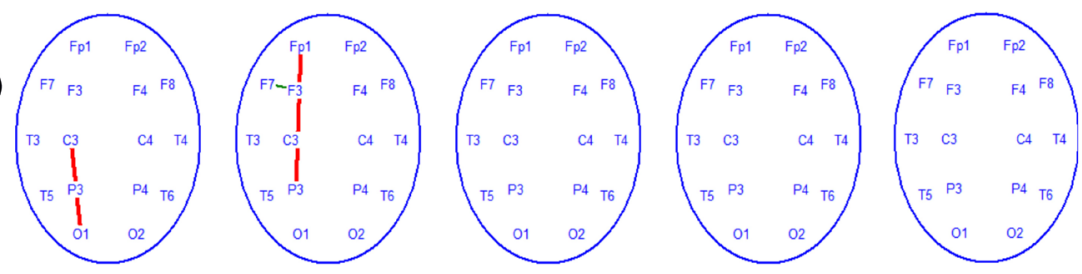

Figure 7. Averaged changes of EEG coherence while performing right-hand (A) and left-hand (B) movements in TBI patients $(\mathrm{n}=9)$ compared to baseline state $(\mathrm{p}<0.01)$. Designations as in Figure 6.

[37] [38]. FMRI responses were observed in the sensorimotor area of the left hemisphere, contralateral to the working hand, medial parts of the superior frontal gyri and ipsilateral cerebellum, while performing motor tasks with the right hand. Hemodynamic reactions to a left-hand task did not have identical topography: there were fewer structures involved in the organization of movements. Left-hand movement produces a greater inter-individual variety of fMRI responses than with the right hand. It was assumed that left-hand movement, which is less common to the right-handed subjects, leads to less structural determinism in response formation [27].

An fMRI analysis of events occurring in the brain of patients with mTBI (without hemiparesis) during hand movements showed changes in comparison with healthy persons, including the involvement of various cerebral structures predominantly at the subcortical levels. A transition to the diffuse form of fMRI response was detected that involved more structures in patients than in the norm. The diffuse form of the brain reaction was reported earlier by other authors, e.g., for motor and spatial tasks in TBI patients [29]. Similar reactive changes took other forms of cerebral pathology, for instance, brain tumors [37], and occurred in patients with speech disorders of different origins [35].

According to fMRI data, comparison of brain structures involved in the execution of the right- and left-handed movements showed a greater inclusion of the subcortical and nonspecific cortical structures in mTBI patients than in healthy persons. Perhaps this reflects the specific activation of compensatory processes necessary to perform motor tasks for this pathology. According to opinion of some authors, the central nervous system has both rigidly fixed and more flexible functional connections with a significant degree of freedom that are activated in pathology; hence, revealed features of reactive rearrangements in TBI might reflect a greater extent of compensatory engagement of the deep cerebral structures. 
It should be noted that the greatest structural and functional changes were in the dominant left hemisphere of mTBI patients. In addition, the results of EEG studies showed the maximum manifestation of pathological signs in the left hemisphere as reactive rearrangements of the slow (delta-theta) frequency ranges in these patients. In accordance with the concept of the subcortical genesis of slow rhythms [39] [40], the EEG data presented further confirm the predominant inclusion of subcortical structures in motor functions after mTBI. In contrast, when healthy people performed motor tasks, reactive restructuring manifested in the high-frequency range of cortical alpha band [27]. This fact is consistent with our and others' research and may indicate the leading role of cortical structures in motor functions in a healthy brain [17]-[25] [29] [30] [31].

Our data on a greater sensitivity of the left hemisphere to brain injury is not an exclusive phenomenon. Other authors' voxel-based morphometry data revealed bilateral grey matter abnormalities and mostly in the left hemisphere, in the regions of the primary motor cortex corresponding to the representation of the articulators, accompanied by speech disorders in persons who had brain trauma as a child [15]. A similar pattern was also reported for patients with strokes that were more likely to appear in the left hemisphere [41].

The left hemisphere is also more sensitive to deleterious environmental factors, including low doses of radiation [41] [42]. It was shown that interhemispheric asymmetry decreased in young Chernobyl victims (45 - 50 y.o.) in comparison with the age-related norm, while it was similar to hemispheric asymmetry in healthy persons of 60 - 65 y.o. These changes occurred mainly due to a decreased functional state of the left hemisphere [42].

Furthermore, healthy right-handed subjects experienced an age-related decrease in brain asymmetry mainly due to a reduced reactivity of the dominant left hemisphere [43]. Cabeza proposed HAROLD (hemispheric asymmetry reduction in elderly people) hypothesis of human brain aging, which explained the aging process mainly by a primary reduction of the left hemispheric functional activity [44].

It is important to note that recent studies have revealed accelerated brain aging in patients with the effects of TBI compared with healthy people [45]. The long-term effects of TBI can resemble those observed in normal ageing, suggesting that TBI may accelerate the ageing process. Considering a neuroimaging model and the data of this study, the authors found a predicted cognitive impairment. These data indicated that brain tissue loss increases throughout the chronic post injury phase, suggesting that TBI accelerates the rate of brain atrophy. There are some empirical data that TBI causes structural brain changes that resemble the atrophy seen during aging [15]. This may be an important factor in the increased susceptibility to dementia and other age-associated conditions in TBI patients, motivating further research into the age-like effects of brain injury and other neurological diseases.

Our and others' research data demonstrate a greater sensitivity of the dominant left hemisphere and cortical structures, i.e. younger brain structures, to 
traumatic effect. Moreover, these results allowed us to explain the brain mechanisms of accelerated brain aging after TBI by a preferential dysfunction of the left hemisphere and cognitive decline in TBI patients described by numerous authors [19] [20].

Thus, a complex estimation of fMRI and EEG reactive changes during motor tasks in right-hand patients with $\mathrm{mTBI}$ suggests that the younger cerebral structures, such as cortical ones, and the dominant left hemisphere are primarily damaged due to a traumatic event.

Further research would require different functional tasks, additional methods for a detailed investigation of structural brain organization and a larger sample of patients to confirm this hypothesis. However, even at this stage one can assume that an increase of fMRI volume and a number of activated brain structures in pathology can reflect activating the compensatory process necessary to execute motor function in patients after mTBI.

\section{Conclusion}

Results of our study allowed us to suggest that in right-handed subjects with mTBI (without hemiparesis), pathological fMRI and EEG responses were expressed mostly in the dominant left hemisphere, while performing right-hand movements. The changes of fMRI and EEG responses covered a large number of brain structures, predominantly of subcortical formations areas, including areas nonspecific for the motor analyser. These results confirm the idea that the younger brain structures, the cortex and left hemisphere, are more sensitive to environmental factors, including those of traumatic genesis.

\section{Acknowledgments}

This study was supported by the RFBR Grant No. 17-06-01012 OGN.

\section{Conflicts of Interest}

The authors declare no conflicts of interest regarding the publication of this paper.

\section{References}

[1] Bigler, E.D. and Wilde, E.A. (2010) Quantitative Neuroimaging and the Prediction of Rehabilitation Outcome Following Traumatic Brain Injury. Frontiers in Human Neuroscience, 4, 228-238. https://doi.org/10.3389/fnhum.2010.00228

[2] Zakharova, N., Kornienko, V., Potapov, A. and Pronin, I. (2014) Neuroimaging of Traumatic Brain Injury. Springer, London, 159 p. https://doi.org/10.1007/978-3-319-04355-5

[3] Marshall, L.F. (2000) Head Injury: Recent Past, Present, and Future. Neurosurgery, 47, 546-557. https://doi.org/10.1227/00006123-200009000-00002

[4] Cherubini, A., Luccichenti, G., Peran, P., Hagberg, G.E., Barba, C., Formisano, R. and Sabatini, U. (2007) Multimodal fMRI Tractography in Normal Subjects and Clinically Recovered Traumatic Brain in Jury Patients. Neuroimage, 34, 1331-1341. https://doi.org/10.1016/j.neuroimage.2006.11.024 
[5] Levine, B., Fujiwara, E., O'Connor, C., et al. (2006) In Vivo Characterization of Traumatic Brain Injury Neuropathology with Structural and Functional Neuroimaging. Journal of Neurotrauma, 23, 1396-1403.

https://doi.org/10.1089/neu.2006.23.1396

[6] Vos, P.E. and Bigler, E.D. (2011) White Matter in Traumatic Brain Injury: Dis- or Dysconnection? Neurology, 77, 810-817. https://doi.org/10.1212/WNL.0b013e31822b015b

[7] Quinn, B. and Sullivan, S.J. (2000) The Identification by Physiotherapists of the Physical Problems Resulting from a Mild Traumatic Brain Injury. Brain Injury, 14, 1063-1068. https://doi.org/10.1080/02699050050203568

[8] Draper, K. and Ponsford, J. (2008) Cognitive Functioning Ten Years Following Traumatic Brain Injury and Rehabilitation. Neuropsychology, 22, 618-625. https://doi.org/10.1037/0894-4105.22.5.618

[9] Kinnunen, K.M., Greenwood, R., Powell, J.H., et al. (2011) White Matter Damage and Cognitive Impairment after Traumatic Brain Injury. Brain, 134, 449-463. https://doi.org/10.1093/brain/awq347

[10] Bigler, E.D. (2013) Traumatic Brain Injury, Neuroimaging and Neurodegeneration. Frontiers in Human Neuroscience, 7, 395-404. https://doi.org/10.3389/fnhum.2013.00395

[11] Sharp, D.J., Beckmann, C.F., Greenwood, R., et al. (2011) Default Mode Network Functional and Structural Connectivity after Traumatic Brain Injury. Brain, 134, 2233-2247. https://doi.org/10.1093/brain/awr175

[12] Ross, D.E. (2011) Review of Longitudinal Studies of MRI Brain Volumetry in Patients with Traumatic Brain Injury. Brain Injury, 25, 1271-1278. https://doi.org/10.3109/02699052.2011.624568

[13] Sidaros, A., Engberg, A., Sidaros, K., et al. (2008) Diffusion Tensor Imaging during Recovery from Severe Traumatic Brain Injury and Relation to Clinical Outcome: A Longitudinal Study. Brain, 131, 559-668. https://doi.org/10.1093/brain/awm294

[14] Sidaros, A., Skimminge, A., Liptrot, M.G., et al. (2008) Long-Term Global and Regional Brain Volume Changes Following Severe Traumatic Brain Injury: A Longitudinal Study with Clinical Correlates. NeuroImage, 44, 1-14.

https://doi.org/10.1016/j.neuroimage.2008.08.030

[15] Cole, J.H., Leech, R. and Sharp, D.J. (2015) Prediction of Brain Age Suggests Accelerated Atrophy after Traumatic Brain Injury. Annals of Neurology, 77, 571-581. https://doi.org/10.1002/ana.24367

[16] Mukhina, T.S., Sharova, E.V., Boldyreva, G.N., et al. (2017) The Neuroanatomy of Active Arm Movements in Patients with Severe Cranio-Cerebral Trauma (Analysis of fMRI Data). Nevrologiya, Neiropsikhiatriya, Psikhosomatika, 9, 27-33.

[17] Bazarizan, J.J., Cernak, I. and Noble-Haeusslein, L. (2009) Long-Term Neurologic Outcomes after Traumatic Brain Injury. The Journal of Head Trauma Rehabilitation, 24, 439-451. https://doi.org/10.1097/HTR.0b013e3181c15600

[18] Jaiswal, N., Ray, W. and Slobounov, S. (2010) Encoding of Visual-Spatial Information in Working Memory Requires More Cerebral Efforts than Retrieval: Evidence from an EEG and Virtual Reality Study. Brain Research, 1347, 80-89. https://doi.org/10.1016/j.brainres.2010.05.086

[19] Slobounov, S., Sebastianellia, W. and Hallett, M. (2012) Residual Brain Dysfunction Observed One Year Post-Mild Traumatic Brain Injury: Combined EEG and Balance Study. Clinical Neurophysiology, 123, 1755-1761.

https://doi.org/10.1016/j.clinph.2011.12.022 
[20] Cao, C. and Slobounov, S. (2010) Alteration of Cortical Functional Connectivity as a Result of Traumatic Brain Injury Revealed by Graph Theory, ICA, and sLORETA Analyses of EEG Signals. IEEE Transactions on Neural Systems and Rehabilitation Engineering, 18, 11-19. https://doi.org/10.1109/TNSRE.2009.2027704

[21] Gao, X., Enikolopov, G. and Chen, J. (2009) Moderate Traumatic Brain Injury Promotes Proliferation of Quiescent Neural Progenitors in the Adult Hippocampus. Experimental Neurology, 219, 516-525. https://doi.org/10.1016/j.expneurol.2009.07.007

[22] Zhang, K., Johnson, B., Penelli, D., Ray, W., Sebastinelly, W. and Slobounov, S. (2010) Are Functional Deficits in Concussed Individuals Consistent with White Matter Structural Alterations: Combined fMRI and DTI Study. Experimental Brain Research, 204, 57-70. https://doi.org/10.1007/s00221-010-2294-3

[23] Barwick, F., Arnett, P. and Slobounov, S. (2012) EEG Correlates of Fatigue during Administration of A Neuropsychological Test Battery. Clinical Neurophysiology, 123, 278-284. https://doi.org/10.1016/j.clinph.2011.06.027

[24] Boldyreva, G.N., Sharova, E.V., Zhavoronkova, L.A., Chelyapina, M.V., Dubrovskaya, L.P., Smirnov, A.S., Troshina, E.M., Pronin, I.N. and Kornienko, V.N. (2013) Structural and Functional Peculiarity of Brain Activity to Performance and Imaginary Motor Tasks in Healthy Persons (EEG and fMRI Study). Zhurnal vysshei nervnoi deiatelnosti imeni I P Pavlova, 63, 316-327.

[25] Babiloni, F., Babiloni, C., Carducci, F., et al. (2003) Multimodal Integration of High-Resolution EEG and Functional Magnetic Resonance Imaging Data: A Simulation Study. NeuroImage, 19, 1-15. https://doi.org/10.1016/S1053-8119(03)00052-1

[26] Babiloni, C., Infarinato, F., Marzano, N., Iacoboni, M., Dassù, F., Soricelli, A., Rossini, P.M., Limatola, C. and Del Percio, P. (2011) Intra-Hemispheric Functional Coupling of Alpha Rhythms Is Related to Golfer's Performance: A Coherence EEG Study. International Journal of Psychophysiology, 121, 260-268.

https://doi.org/10.1016/j.ijpsycho.2011.09.008

[27] Babiloni, C., Del Percio, C., Arendt-Nelsen, L., Soricelli, A., Rossini, G.L. and Capotosto, P. (2014) Cortical EEG Alpha Rhythms Reflect Task-Specific Somatosensory and Motor Interactions in Human. Clinical Neurophysiology, 125, 1936-1945. https://doi.org/10.1016/j.clinph.2014.04.021

[28] Shtark, M.B., Korostyshevskaya, A.M., Rezakova, M.V. and Savelov, A.A. (2012) Functional Magnetic Resonance Imaging and Neuroscience. Uspekhi Fiziologicheskikh Nauk, 43, 324-335.

[29] Boldyreva, G.N., Sharova, E.V., Zhavoronkova, L.A., Chelyapina, M.V., Dubrovskaya, L.P., Smirnov, A.S., Troshina, E.M., Pronin, I.N. and Kornienko, V.N. (2015) Comparison of fMRI Brain Responses in Healthy Subjects While Active, Passive and Imagined Hand Movements. Medical Visualization, 5, 100-110.

[30] Zhavoronkova, L.A., Boldyreva, G.N., Kuptsova, S.V., Sharova, E.V., Smirnov, A.S. and Proninc, I.N. (2017) fMRI Responses of the Brain during Active and Passive Movements in Left-Handed Subjects. Human Physiology, 43, 191-198. https://doi.org/10.1134/S0362119717010108

[31] Sharova, E.V., Gavron, A.A., Abdulaev, A.A., Smirnov, A.S., Fadeeva, L.M., Chelyapina, M.V., Zhavoronkova, L.A., Boldyreva, G.N., Kulikov, M.A., Verkhlyutov, V.M., Pronin, I.N. and Kornienko, V.N. (2015) Our Experience of the fMRI Resting State (RS) Analysis in Normal Subjects According to the FSL Software. Medical Visualization, 4, 6-17.

[32] Mulert, C. and Lemieux, L. (2010) EEG-fMRI Physiological Basis, Echnique and 
Applications. Springer-Verlag, Berlin, 115. https://doi.org/10.1007/978-3-540-87919-0

[33] Boldyreva, G.N., Zhavoronkova, L.A., Sharova, E.V., et al. (2009) FMRI-EEG Study of Healthy Human Brain Responses to Functional Loads. Human Physiology, 35, 274-284. https://doi.org/10.1134/S0362119709030037

[34] Sharova, E.V., Migalev, A.S., Kulikov, M.A., et al. (2012) Comparison of Reactive EEG Changes and fMRI-Characteristics of the Brain of a Healthy Person on the Basis of Multidimensional Statistics. Zhurnal vysshei nervnoi deiatelnosti imeni I P Pavlova, 62, 143-153.

[35] Zhang, K., Johnson, B., Pennell, D., et al. (2010) Are Functional Deficits in Concussed Individuals Consistent with White Matter Structural Alterations: Combined FMRI \& DTI Study. Experimental Brain Research, 204, 57-68. https://doi.org/10.1007/s00221-010-2294-3

[36] Boldyreva, G.N., Zhavoronkova, L.A., Sharova, E.V., et al. (2012) EEG-fMRT Study Human Brain Functional Specialization in Healthy Persons and Patients with Cerebral Pathology. Medical Visualization, 1, 15-25.

[37] Boldyreva, G.N., Sharov, E.V., Zhavoronkova, L.A., Chelyapina, M.V., Dubrovinskaia, L.P., Simonova, O.A., Smirnov, A.S., Troshina, E.M. and Korniyenko, V.N. (2014) EEG and fMRI Reactions of a Healthy Brain at Active and Passive Movements by Loading Hand. Zhurnal vysshei nervnoi deiatelnosti imeni I P Pavlova, 64, 488-499.

[38] Sclocco, R., Tana, M.G., Viosani, E., Gilioli, I., Panzica, F., Franceschetti, S., Gerutti, S. and Bianchi, A.M. (2014) EEG-Informed fMRI Analysis a Hand Grip Task: Estimating the Relationship between EEG Rhythms and the BOLD Signal. Frontiers in Human Neuroscience, 8, 186. https://doi.org/10.3389/fnhum.2014.00186

[39] Lopez da Silva, F.H. (1991) Neural Mechanisms Underlying Brain Waves: From Neural Membrane to Networks. EEG and Clinical Neurophysiology, 79, 81. https://doi.org/10.1016/0013-4694(91)90044-5

[40] Buzsaki, G. (2006) Rhythms of the Brain. Oxford University Press, Oxford, 215. https://doi.org/10.1093/acprof:oso/9780195301069.001.0001

[41] Bragina, N.N. and Dobrokhotova, T.A. (1988) Funktsional'nye asimmetrii cheloveka (Functional Asymmetries in Humans). Meditsina, Moscow, 120.

[42] Zhavoronkova, L.A., Belostotskii, A.P., Kholodova, N.B., et al. (2013) Impairments to Higher Mental Functions and Cognitive Auditory Evoked Potentials in Chernobyl Clean-Up Workers. Neuroscience and Behavioral Physiology, 43, 887-896. https://doi.org/10.1007/s11055-013-9824-y

[43] Zhavoronkova, L.A. (2019) Neurophysiology: Interhemispheric Asymmetry of the Human Brain (Right-Handed and Left-Handed). Yuwrite, Moscow, 217 p.

[44] Cabeza, R. (2002) Cognitive Neuroscience of Aging: Contributions of Functional Neuroimaging. Scandinavian Journal of Psychology, 42, 277-286. https://doi.org/10.1111/1467-9450.00237

[45] Imeria, A., Suzuki, S., Rostowsky, K., Maher, A., Chowdhury, N. and Van Horn, J. (2018) Effects of Cerebrovascular Micropathology upon Cognitive Impairments and Brain Aging Trajectories Subsequent to Geriatric Brain Injury. International Journal of Psychophysiology, 131, S29. https://doi.org/10.1016/j.ijpsycho.2018.07.091 